\title{
Teratoma with Somatic-Type Malignancy
}

National Cancer Institute

\section{Source}

National Cancer Institute. Teratoma with Somatic-Type Malignancy. NCI Thesaurus.

Code C4289.

A teratoma which is characterized by morphologic transformation to malignancy and an aggressive clinical course. The malignant component most often is sarcomatous or carcinomatous. 